\title{
Pinning Synchronization of Switched Complex Dynamical Networks
}

\author{
Liming Du, ${ }^{1,2}$ Feng Qiao, ${ }^{2}$ and Fengying Wang ${ }^{2}$ \\ ${ }^{1}$ State Key Laboratory of Synthetical Automation for Process Industries, College of Information Science \& Engineering, \\ Northeastern University, Shenyang 110819, China \\ ${ }^{2}$ Faculty of Information \& Control Engineering, Shenyang Jianzhu University, Shenyang 110168, China
}

Correspondence should be addressed to Liming Du; duliming_666@163.com

Received 15 August 2014; Revised 8 October 2014; Accepted 28 October 2014

Academic Editor: Xi-Ming Sun

Copyright (C) 2015 Liming Du et al. This is an open access article distributed under the Creative Commons Attribution License, which permits unrestricted use, distribution, and reproduction in any medium, provided the original work is properly cited.

\begin{abstract}
Network topology and node dynamics play a key role in forming synchronization of complex networks. Unfortunately there is no effective synchronization criterion for pinning synchronization of complex dynamical networks with switching topology. In this paper, pinning synchronization of complex dynamical networks with switching topology is studied. Two basic problems are considered: one is pinning synchronization of switched complex networks under arbitrary switching; the other is pinning synchronization of switched complex networks by design of switching when synchronization cannot achieved by using any individual connection topology alone. For the two problems, common Lyapunov function method and single Lyapunov function method are used respectively, some global synchronization criteria are proposed and the designed switching law is given. Finally, simulation results verify the validity of the results.
\end{abstract}

\section{Introduction}

A complex dynamical network is a set of coupled nodes interconnected by edges, in which each node is a dynamical system $[1,2]$. Undoubtedly, many systems in nature can be described by models of complex networks, such as ecological networks, power grids, wireless communication networks, and World Wide Web. Over the last decade, the analysis and control of dynamical networks have become research focus in many fields, such as mathematics, physics, biology, and engineering.

Synchronization, the most important collective behavior of complex dynamical networks, has received much of the focus [3-5]. The synchronization of complex dynamical networks has been extensively investigated and many synchronization criteria for complex networks have been proposed, such as the master stability function based criteria [6], Lyapunov function based criteria $[7,8]$, and graph stability method based criteria [9].
As we know now, some complex network can be synchronized by itself, but the general case is that the whole network cannot synchronize by itself; from the perspective of control theory, designing controllers is an effective method, and one natural idea is to assign controllers to all nodes of the controlled complex dynamical network [10-12].

However, complex networks in the real world normally have a large number of nodes. Therefore, it is usually difficult to control a complex network by adding the controllers to all nodes. To reduce the number of the controllers, a natural approach is to control a complex network by pinning part of nodes. This idea is actually adding the controllers to a small fraction of network nodes to achieve synchronization of the whole complex networks. In [13], Grigoriev et al. investigated the pinning control of spatiotemporal chaos. Subsequently, Parekh et al. studied the local and global control in coupled map lattices [14]. In $[15,16]$, Wang and Chen considered the problem of pinning a complex dynamical network to its equilibrium, and both specific and random pinning strategies 
were proposed. In [17], the controllability of a coupled complex network via pinning has been studied by means of a Master Stability Function approach. In [18], Chen et al. pointed out that a general complex network can be pinned by a single controller if the coupling strength is large enough. A network under a typical framework can realize synchronization subject to any linear feedback pinning scheme and the relationship between coupling strength and the number of pinning nodes for a general complex dynamical network through adaptive pinning $[19,20]$.

Note that most investigations about synchronization of complex networks under pinning control are with the constant connection topology. Actually, constant connection is only a special case. It is well known that the interaction of two different nodes in the real world networks always evolves with time continuously, and the interaction between different nodes may change abruptly at some time instants, which results in switching topology, such as mobile agents [21] and power grids [22].

The switched system theory provides an effective tool for studying complex networks with switching topology [23]. By using the common Lyapunov function method, the synchronization problem for complex dynamical networks with switching topology has been studied in [24]. In [25], the synchronization of switched complex dynamical networks was discussed from the view point of switched systems, under the assumptions that all the connection outer matrices are simultaneous triangularization, and several synchronization criteria have been established by means of constructing a common Lyapunov function and single Lyapunov and multiple Lyapunov functions, respectively. In [26], the synchronization problem was studied with switched coupling using the average dwell time method. A synchronization criterion for dynamical networks with nonidentical nodes and switching topology was given in [27]. However, to our knowledge, so far, none of the results of pinning synchronization of complex networks with switching topology have been reported. Motivated by the above discussions, in this paper, the pinning synchronization of complex dynamical networks is investigated with switching topology. First, we study the problem of pinning synchronization of switched complex network under arbitrary switching. Second, when pinning synchronization is impossible for each individual connection topology, the problem of synchronization via the design of switching signal of pregiven connection topology is studied. By using common Lyapunov function method and single Lyapunov function method, respectively, we design controllers and the switching laws to ensure the pinning synchronization. Without assuming that the coupling matrix is symmetric, we give some criteria for the global pinning synchronization of complex networks with switching topology.

The remainder of the paper is organized as follows. Some preliminaries are described in Section 2. Pinning synchronization criteria for arbitrary switching are derived in Section 3. In Section 4, pinning synchronization via design of switching is studied. In Section 5, several numerical examples are given. Conclusions are drawn in Section 6.

\section{Preliminaries}

Now consider a switched complex dynamical network with $N$ identical nodes; the mathematics model of the system can be described as follows:

$$
\dot{x}_{i}=f\left(x_{i}\right)+c \sum_{j=1}^{N} a_{i j}^{\sigma(t)} \Gamma x_{j}, \quad i=1,2, \ldots, N,
$$

where $x_{i}=\left(x_{i 1}, x_{i 2}, \ldots, x_{i n}\right)^{T} \in R^{n}$ is the state vector of the $i$ th node, $\Gamma$ is the inner-coupling matrix between two connected nodes, $f$ is a continuously differentiable vector function, $c$ is the coupling strength, $\sigma:[0, \infty) \rightarrow M=\{1,2, \ldots, m\}$, for each $k \in M, A_{k}=\left\{a_{i j}^{k}\right\} \in R^{N \times N}$ is the outer-coupling matrix, assume $a_{i i}^{k}=\sum_{j=1, j \neq i}^{N} a_{i j}^{k}$, and let $s(t)$ be a solution of each isolated node; that is, $\dot{\boldsymbol{s}}(t)=f(s(t))$.

Normally, a complex dynamical network has a large number of nodes, so it is difficult to add the controllers to all nodes. By pinning control methods, the controllers are to be added to part of nodes, and the other nodes need not be controlled; without loss of generality, let the first $l$ nodes be controlled; then (1) can be expressed as

$$
\begin{aligned}
& \dot{x}_{i}=f\left(x_{i}\right)+c \sum_{j=1}^{N} a_{i j}^{\sigma(t)} \Gamma x_{j}+u_{i}^{\sigma(t)}, \quad i=1,2, \ldots, l, \\
& \dot{x}_{i}=f\left(x_{i}\right)+c \sum_{j=1}^{N} a_{i j}^{\sigma(t)} \Gamma x_{j}, \quad i=l+1, l+2, \ldots, N,
\end{aligned}
$$

where $u_{i}^{k}$ are the given controller, which can be described as

$$
u_{i}^{k}=-c q_{i}^{k} \Gamma\left(x_{i}-s\right), \quad i=1,2, \ldots, l, q_{i}^{k}>0 .
$$

It is easy to see that the systems (2) are pinned to synchronization if $\lim _{t \rightarrow \infty}\left\|x_{i}(t)-s(t)\right\|=0$. Next, define $e_{i}=x_{i}-s$; then the error system can be described as

$$
\begin{gathered}
\dot{e}_{i}=f\left(x_{i}\right)-f(s)+c \sum_{j=1}^{N} a_{i j}^{\sigma(t)} \Gamma e_{j}-c q_{i}^{\sigma(t)} \Gamma e_{i}, \quad i=1,2, \ldots, l, \\
\dot{e}_{i}=f\left(x_{i}\right)-f(s)+c \sum_{j=1}^{N} a_{i j}^{\sigma(t)} \Gamma e_{j}, \quad i=l+1, l+2, \ldots, N,
\end{gathered}
$$

and it should be noted that only the first $l$ nodes have controllers, so the last term of the second line of (4) is zero. Now, the synchronization problem of system (2) turns into the stability of system (4); throughout this paper, we need the following assumption.

Assumption 1. Assume that there is a positive defined matrix $P$ and a constant matrix $K$, such that $f$ satisfies the following inequality:

$$
\begin{array}{r}
(x-y)^{T} P(f(x)-f(y)) \leq(x-y)^{T} K \Gamma(x-y), \\
\forall x, y \in R^{n} .
\end{array}
$$


Note that Assumption 1 is very mild; many systems satisfy this condition, such as Lorenz system, Liu system, and Chen system. In the following section, we will investigate the problems of pinning synchronization by common Lyapunov function method and single Lyapunov function method, respectively.

\section{Arbitrary Switching}

In this section, we study the pinning synchronization of complex dynamical networks under arbitrary switching topology. A network with switching topology may not realize synchronization by pinning controller, even if pinning synchronization is achieved by using each individual connection topology alone. Therefore, seeking for pinning synchronization criteria for arbitrary switching topology is not trivial. By using common Lyapunov function method, a criterion is derived to ensure the global synchronization under arbitrary switching.

Theorem 2. Suppose that Assumption 1 holds, the pinning controlled complex dynamical system in (2) achieves global synchronization under arbitrary switching if there exist a positive definite matrix $P$ such that the condition

$$
I_{N} \otimes K \Gamma+c\left(A_{k}-Q_{k}\right) \otimes P \Gamma<0, \quad k=1,2, \ldots, m,
$$

holds, where $\otimes$ is Kronecker product, $I_{N}$ is an $N$-dimensional identity matrix, and $Q_{k}=\operatorname{diag}\left\{q_{1}^{k}, q_{2}^{k}, \ldots, q_{l}^{k}, 0, \ldots, 0\right\} \in$ $R^{N \times N}$.

Proof. Construct the common Lyapunov function as the form of

$$
V(t)=\frac{1}{2} \sum_{i=1}^{N} e_{i}^{T} P e_{i} .
$$

The derivative of $V(t)$ along the trajectories of each subsystem of (4) gives

$$
\begin{aligned}
\dot{V}(t) & =\sum_{i=1}^{N} e_{i}^{T} P \dot{e}_{i} \\
& =\sum_{i=1}^{N} e_{i}^{T} P\left[f\left(x_{i}\right)-f(s)+c \sum_{j=1}^{N} a_{i j}^{k} \Gamma e_{i}\right]-c \sum_{i=1}^{l} q_{i}^{k} e_{i}^{T} P \Gamma e_{i} \\
& \leq \sum_{i=1}^{N} e_{i}^{T}\left[K \Gamma e_{i}+c P \sum_{j=1}^{N} a_{i j}^{k} \Gamma e_{i}\right]-c \sum_{i=1}^{l} q_{i}^{k} e_{i}^{T} P \Gamma e_{i} \\
& =e^{T}\left[I_{N} \otimes K \Gamma+c\left(I_{N} \otimes P\right)\left(A_{k} \otimes \Gamma\right)-c\left(Q_{k} \otimes P \Gamma\right)\right] e \\
& =e^{T}\left[I_{N} \otimes K \Gamma+c\left(A_{k} \otimes P \Gamma\right)-c\left(Q_{k} \otimes P \Gamma\right)\right] e,
\end{aligned}
$$

where $e=\left(e_{1}^{T}, e_{2}^{T}, \ldots, e_{N}^{T}\right)^{T}$. From (6), it is easy to see that

$$
\dot{V}(t)<0 \text {, }
$$

the switched complex network in (2) achieves global synchronization with the pinning controller (3) under arbitrary switching, which completes the proof.
Remark 3. For the special case is that $P=I$ and let the network has only one connection topology; then the synchronization condition (6) degenerates into

$$
I_{N} \otimes K \Gamma+c(A-Q) \otimes \Gamma<0,
$$

which is expressed in [20].

Remark 4. If $\Gamma$ is a positive definite matrix and $P$ is replaced with $I$, then the conditions (6) in Theorem 2 can be simplified as

$$
\theta I_{N}+c\left(A_{k}-Q_{k}\right)<0, \quad k=1,2, \ldots, m,
$$

where $\theta=\|K\|$.

\section{Switching Design}

In this section, we will study the case that none of subnetworks can bring pinning synchronization if each individual connection topology is put in use alone. So pinning synchronization of switched networks under arbitrary switching is impossible to be achieved for this case. But pinning synchronization may still be achieved by switching between connection topologies, so we discuss how to realize synchronization by the suitable design of switching ruler between connection topologies and give a convex combination based method.

Let $\bar{A}=\sum_{k=1}^{m} \varepsilon_{k} A_{k}$ with $\varepsilon_{k} \geq 0$ and $\sum_{k=1}^{m} \varepsilon_{k}=1$; then the convex combination of system (2) and the convex combination of error system (3) can be described as

$$
\begin{gathered}
\dot{x}_{i}=f\left(x_{i}\right)+c \sum_{j=1}^{N} \bar{a}_{i j} \Gamma x_{j}+\bar{u}_{i}, \quad i=1,2, \ldots, l, \\
\dot{x}_{i}=f\left(x_{i}\right)+c \sum_{j=1}^{N} \bar{a}_{i j} \Gamma x_{j}, \quad i=l+1, l+2, \ldots, N, \\
\dot{e}_{i}=f\left(x_{i}\right)-f(s)+c \sum_{j=1}^{N} \bar{a}_{i j} \Gamma e_{j}-c \bar{q}_{i} \Gamma e_{i}, \quad i=1,2, \ldots, l, \\
\dot{e}_{i}=f\left(x_{i}\right)-f(s)+c \sum_{j=1}^{N} \bar{a}_{i j} \Gamma e_{j}, \quad i=l+1, l+2, \ldots, N,
\end{gathered}
$$

where $\bar{a}_{i j}=\sum_{k=1}^{m} \varepsilon_{k} a_{i j}, \bar{u}_{i}=\sum_{k=1}^{m} \varepsilon_{k} u_{i}, \bar{q}_{i}=\sum_{k=1}^{m} \varepsilon_{k} q_{i}$, and $\sum_{k=1}^{m} \varepsilon_{k}=1$.

Theorem 5. Suppose that Assumption 1 holds, and there exist a positive definite matrix $P$, and some convex combination coefficients $\varepsilon_{i}$, which satisfies the following condition:

$$
\begin{array}{r}
I_{N} \otimes K \Gamma+c \sum_{k=1}^{m} \varepsilon_{k}\left(A_{k}-Q_{k}\right) \otimes P \Gamma<0, \\
k=1,2, \ldots, m, \quad \sum_{k=1}^{m} \varepsilon_{k}=1,
\end{array}
$$


where $\otimes$ is Kronecker product, $I_{N}$ is an $\mathrm{N}$-dimensional identity matrix, and $Q_{k}=\operatorname{diag}\left\{q_{1}^{k}, q_{2}^{k}, \ldots, q_{l}^{k}, 0, \ldots, 0\right\} \in R^{N \times N}$; then the controlled complex dynamical system in (13) achieves global pinning synchronization under the following switching laws:

$$
\begin{gathered}
\sigma(t)=i \quad \text { if } \sigma\left(t^{-}\right)=i, e \in \Omega_{i}(t), \\
\sigma(t)=j \quad \text { if } \sigma\left(t^{-}\right)=i, e \in \partial \Omega_{i}(t) \cap \Omega_{j}(t),
\end{gathered}
$$

with

$$
\begin{aligned}
& \Omega_{i}(t) \\
& \quad=\left\{e \mid e^{T}\left[I_{N} \otimes K \Gamma+c\left(A_{i} \otimes P \Gamma\right)-c\left(Q_{i} \otimes P \Gamma\right)\right] e<0\right\}, \\
& \Omega_{j}(t) \\
& \quad=\left\{e \mid e^{T}\left[I_{N} \otimes K \Gamma+c\left(A_{j} \otimes P \Gamma\right)-c\left(Q_{j} \otimes P \Gamma\right)\right] e<0\right\}, \\
& \partial \Omega_{i}(t) \\
& \quad=\left\{e \mid e^{T}\left[I_{N} \otimes K \Gamma+c\left(A_{i} \otimes P \Gamma\right)-c\left(Q_{i} \otimes P \Gamma\right)\right] e=0\right\},
\end{aligned}
$$

where $i, j \in\{1,2, \ldots, m\}$.

Proof. Construct the Lyapunov function as the form of

$$
V(t)=\frac{1}{2} \sum_{i=1}^{N} e_{i}^{T} P e_{i}
$$

The derivative of $V(t)$ along the trajectories of system (12) gives

$$
\begin{aligned}
\dot{V}(t) & =\sum_{i=1}^{N} e_{i}^{T} P \dot{e}_{i} \\
& =\sum_{i=1}^{N} e_{i}^{T} P\left[f\left(x_{i}\right)-f(s)+c \sum_{j=1}^{N} \bar{a}_{i j} \Gamma e_{i}\right]-c \sum_{i=1}^{l} \bar{q}_{i} e_{i}^{T} P \Gamma e_{i} \\
& \leq \sum_{i=1}^{N} e_{i}^{T}\left[K \Gamma e_{i}+c P \sum_{j=1}^{N} \bar{a}_{i j} \Gamma e_{i}\right]-c \sum_{i=1}^{l} \bar{q}_{i} e_{i}^{T} P \Gamma e_{i} \\
& =e^{T}\left[I_{N} \otimes K \Gamma+c\left(I_{N} \otimes P\right)(\bar{A} \otimes \Gamma)-c(\bar{Q} \otimes P \Gamma)\right] e \\
& =e^{T}\left[I_{N} \otimes K \Gamma+c(\bar{A} \otimes P \Gamma)-c(\bar{Q} \otimes P \Gamma)\right] e,
\end{aligned}
$$

where $e=\left(e_{1}^{T}, e_{2}^{T}, \ldots, e_{N}^{T}\right)^{T}, \bar{A}=\sum_{k=1}^{m} \varepsilon_{k} A_{k}$, and $\bar{Q}=$ $\sum_{k=1}^{m} \varepsilon_{k} Q_{k}$. If the condition in (14) is satisfied, then the sets $\Omega_{i}(t)$ in (16) will make a partition of $R^{N n}$ by the convex combination technique [23]; that is, $\cup_{i=1}^{m} \Omega_{i}=R^{N n}$. According to the switching laws (16), if the $k$ th subsystem is activated, then the $k$ th subsystem is described as

$$
\begin{aligned}
& \dot{e}_{i}=f\left(x_{i}\right)-f(s)+c \sum_{j=1}^{N} a_{i j}^{k} \Gamma x_{j}-c q_{i}^{k} \Gamma e_{i}, \quad i=1,2, \ldots, l, \\
& \dot{e}_{i}=f\left(x_{i}\right)-f(s)+c \sum_{j=1}^{N} a_{i j}^{k} \Gamma x_{j}, \quad i=l+1, l+2, \ldots, N .
\end{aligned}
$$

The derivative of $V(t)$ along the trajectories of system (19) is

$$
\begin{aligned}
\dot{V}(t) & =\sum_{i=1}^{N} e_{i}^{T} P \dot{e}_{i} \\
& =\sum_{i=1}^{N} e_{i}^{T} P\left[f\left(x_{i}\right)-f(s)+c \sum_{j=1}^{N} a_{i j}^{k} \Gamma e_{i}\right]-c \sum_{i=1}^{l} q_{i}^{k} e_{i}^{T} P \Gamma e_{i} \\
& \leq \sum_{i=1}^{N} e_{i}^{T}\left[K \Gamma e_{i}+c P \sum_{j=1}^{N} a_{i j}^{k} \Gamma e_{i}\right]-c \sum_{i=1}^{l} q_{i}^{k} e_{i}^{T} P \Gamma e_{i} \\
& =e^{T}\left[I_{N} \otimes K \Gamma+c\left(I_{N} \otimes P\right)\left(A_{k} \otimes \Gamma\right)-c\left(Q_{k} \otimes P \Gamma\right)\right] e \\
& =e^{T}\left[I_{N} \otimes K \Gamma+c\left(A_{k} \otimes P \Gamma\right)-c\left(Q_{k} \otimes P \Gamma\right)\right] e<0 .
\end{aligned}
$$

From single Lyapunov function method [23], the complex networks in (2) achieve global pinning synchronization under the switching law (15), which completes the proof.

Remark 6. Similar to Remark 4, if $\Gamma$ is a positive definite matrix and $P$ is replaced with $I$, then the conditions (14) in Theorem 5 can be simplified as

$$
\theta I_{N}+c \sum_{k=1}^{m} \varepsilon_{k}\left(A_{k}-Q_{k}\right)<0, \quad \sum_{k=1}^{m} \varepsilon_{k}=1,
$$

where $\theta=\|K\|$.

Remark 7. Although (7) and (17) are the same expression, these equations have different meanings. Equation (7) has been used as common Lyapunov function, which needs $\dot{V}(t)<0$ for all the subnetworks. It means that each pinning controlled subnetwork can achieve synchronization. However, (17) has been used as single Lyapunov function, compared with common Lyapunov function; it only needs $\dot{V}(t)<0$ for the time period when the subnetwork is activated; it is easy to see that single Lyapunov function method can deal with more general case of switched complex networks.

Remark 8. Compared with [25], there are three distinct features. First of all, we investigated the pinning synchronization problems of switched complex networks, including pinning controller and switching laws design, however, [25] only considering the synchronization problems of complex networks from the switched system point of view, and it 
does not deal with the design of controller. So far, no synchronization criteria have been reported for dynamical networks with switching topology via pinning controller, so the research topic is significant. Secondly, the assumptions in the two papers are different. The results of [25] are under the assumptions that all the connection outer matrices are simultaneous triangularization, and these assumptions are not easily satisfied. Compared with it, this paper does not need these assumptions. Therefore, the results of this paper cover more general cases of the dynamical networks in the real world. Thirdly, compared with [25], the result of this paper is more simpler and is easy to be verified, which is conducive to engineering applications.

\section{Examples}

In this section, two simple examples are used to explain the effectiveness of the proposed network synchronization criteria.

Example 1. Consider the network

$$
\begin{gathered}
\dot{x}_{i}=f\left(x_{i}\right)+c \sum_{j=1}^{N} a_{i j}^{k} \Gamma x_{j}+u_{i}^{k}, \quad i=1,2, \ldots, l, k=1,2 \\
\dot{x}_{i}=f\left(x_{i}\right)+c \sum_{j=1}^{N} a_{i j}^{k} \Gamma x_{j}, \quad i=l+1, l+2, \ldots, N, k=1,2,
\end{gathered}
$$

where

$$
f\left(x_{i}\right)=\left\{\begin{array}{l}
\dot{x}_{i 1}=10\left(x_{i 2}-x_{i 1}\right), \\
\dot{x}_{i 2}=40 x_{i 1}-25 x_{i 1} x_{i 3}, \\
\dot{x}_{i 3}=-x_{i 3}+4 x_{i 1}^{2} .
\end{array}\right.
$$

Let $T=\operatorname{diag}(1,2,1), c=6$; herein we assume that the network structure of (21) obeys the scale-free distribution of the BA model [27]; the first subsystem's parameters are given by $N=30, m_{0}=m=3$, and the second subsystem's parameters are given by $N=30, m_{0}=m=5$, and, without loss of generality, the pinning controllers are added to the first node. Let $q_{1}^{1}=q_{1}^{2}=20$; the conditions of Theorem 2 are satisfied. The states of error $e_{i}(i=1,2, \ldots, 30)$ are illustrated in Figures 1, 2, and 3 with switching signal in Figure 4, which show that the controlled network is globally asymptotically stable under arbitrary switching by the pinning controller (3).

Example 2. Let $f$ and $\Gamma$ be the same as (21), and the pinning controllers are added to the first node. The outer-coupling matrices $A_{1}$ and $A_{2}$ are pregiven; let $q_{1}^{1}=q_{1}^{2}=90$; then the states of error $e_{i}(i=1,2, \ldots, 30)$ are illustrated in Figures 5, $6,7,8,9$, and 10 .

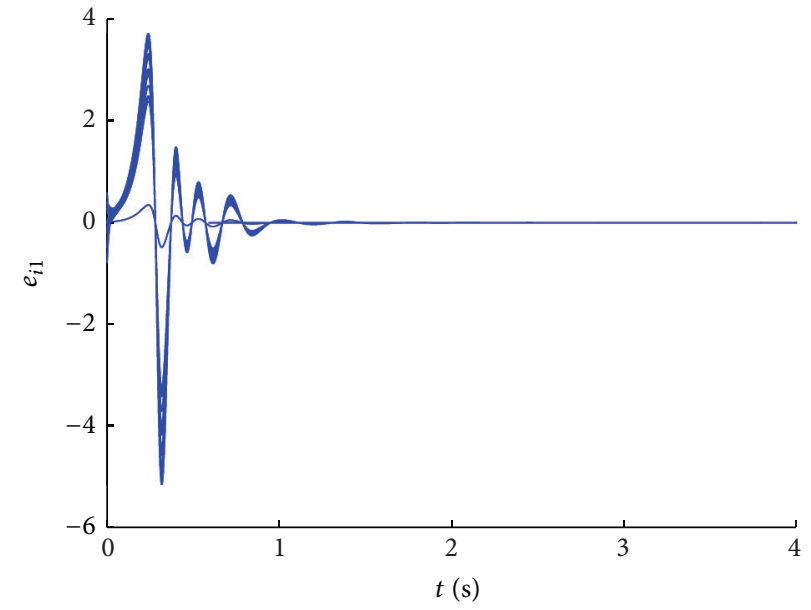

FIgURE 1: The synchronization errors $e_{i 1}$ of complex dynamical system (22).

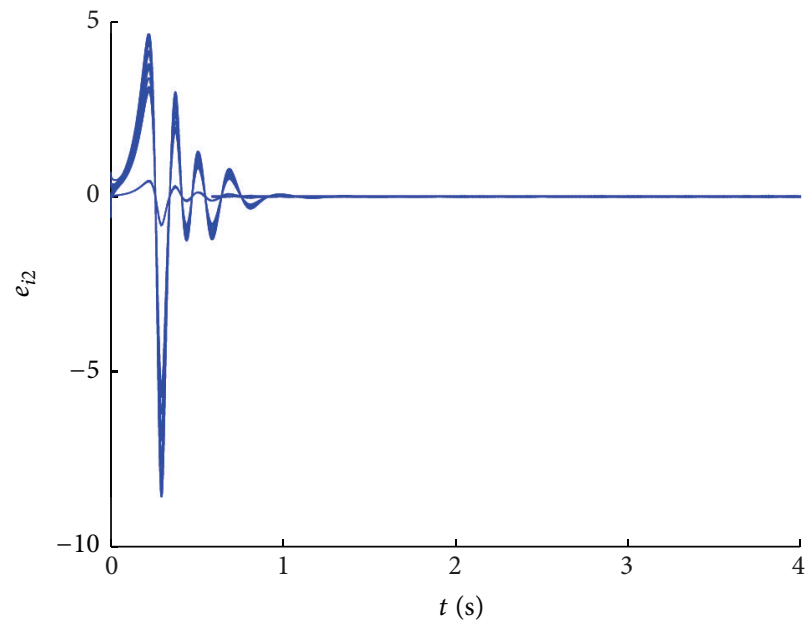

FIGURE 2: The synchronization errors $e_{i 2}$ of complex dynamical system (22).

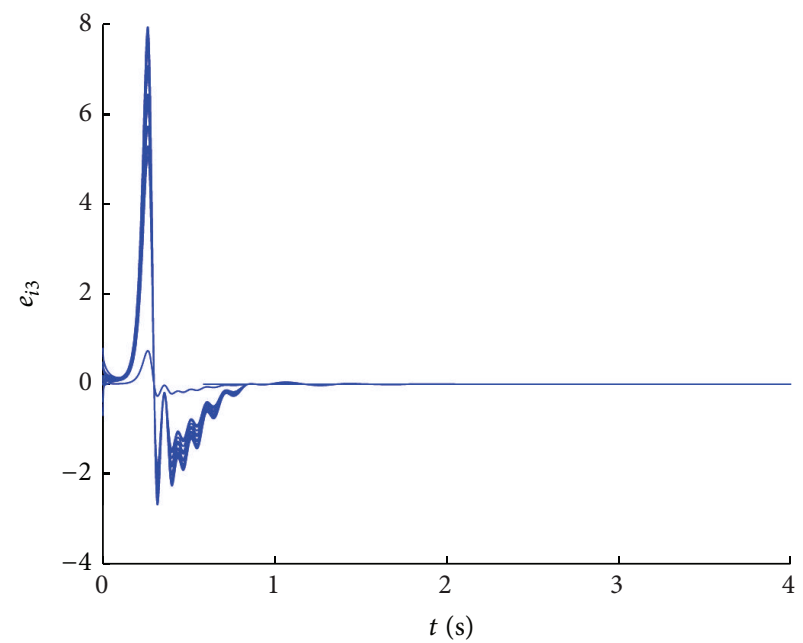

FIGURE 3: The synchronization errors $e_{i 3}$ of complex dynamical system (22). 


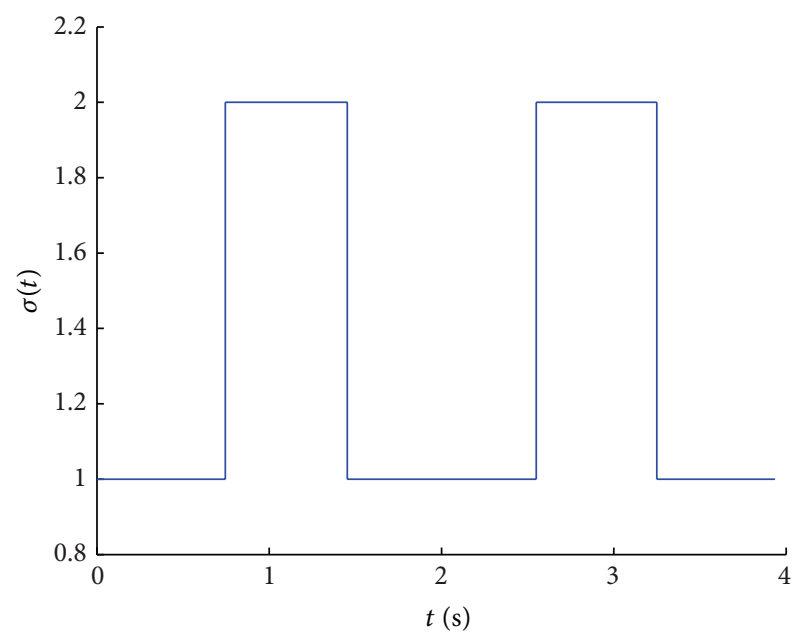

FIGURE 4: The switching signal of system (22).

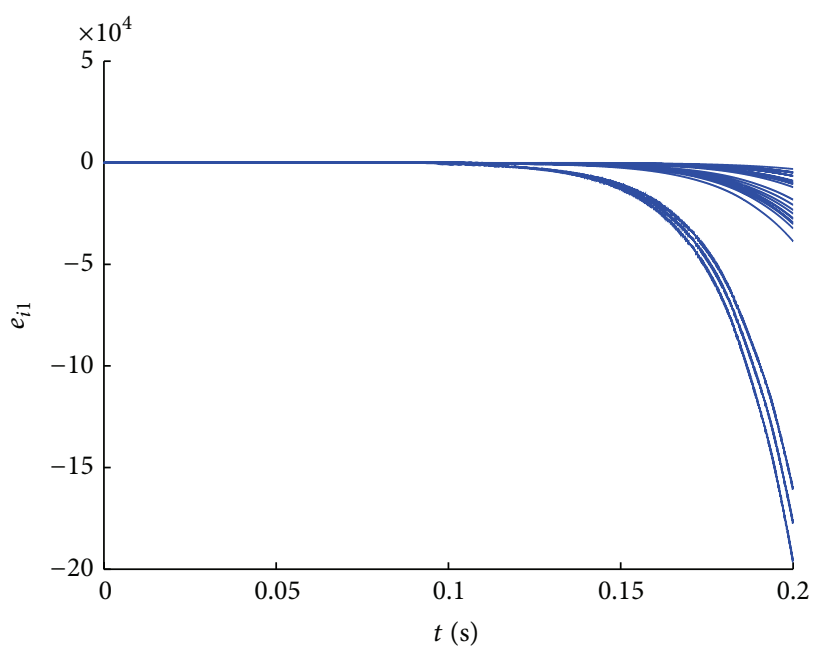

Figure 5: The synchronization errors $e_{i 1}$ of the subnetwork 1 of Example 2.

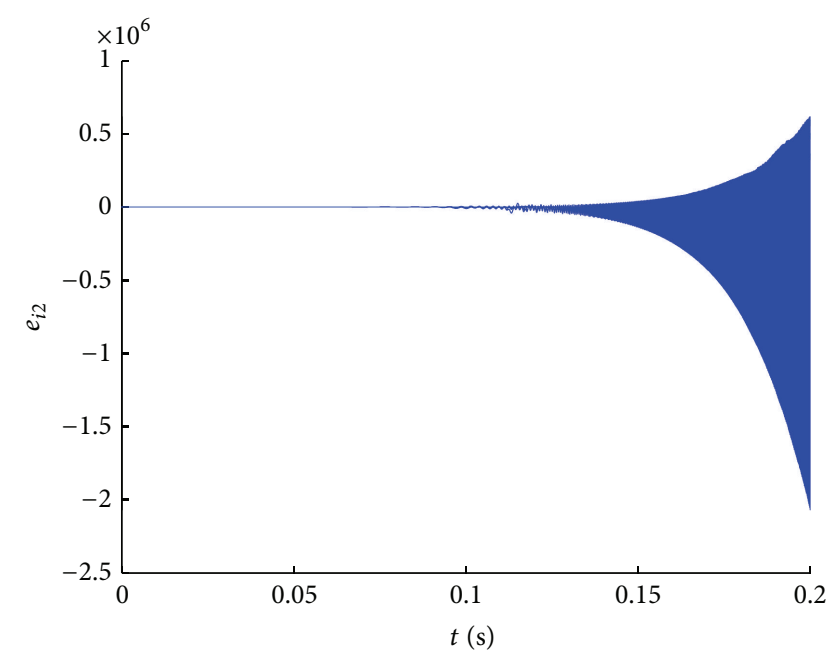

FIGURE 6: The synchronization errors $e_{i 2}$ of the subnetwork 1 of Example 2.

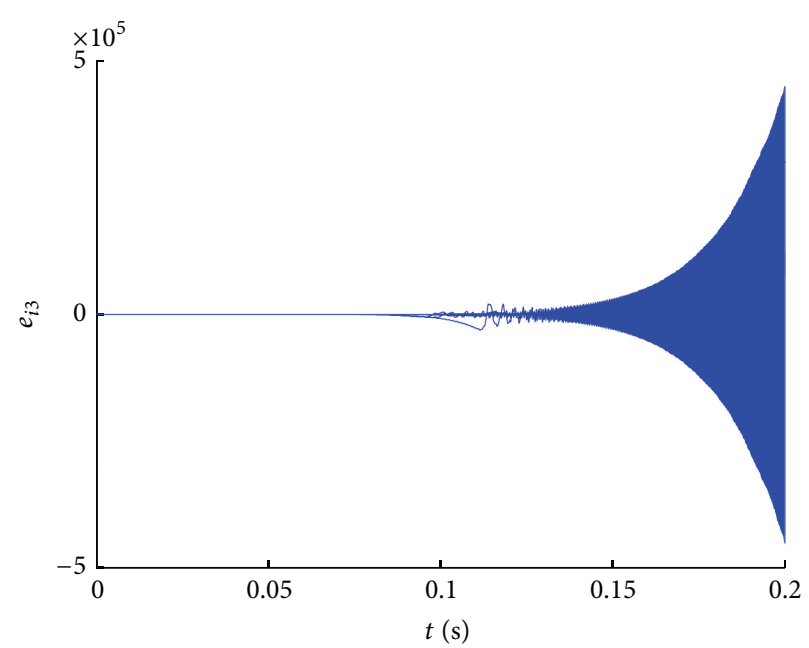

FIGURE 7: The synchronization errors $e_{i 3}$ of the subnetwork 1 in Example 2.

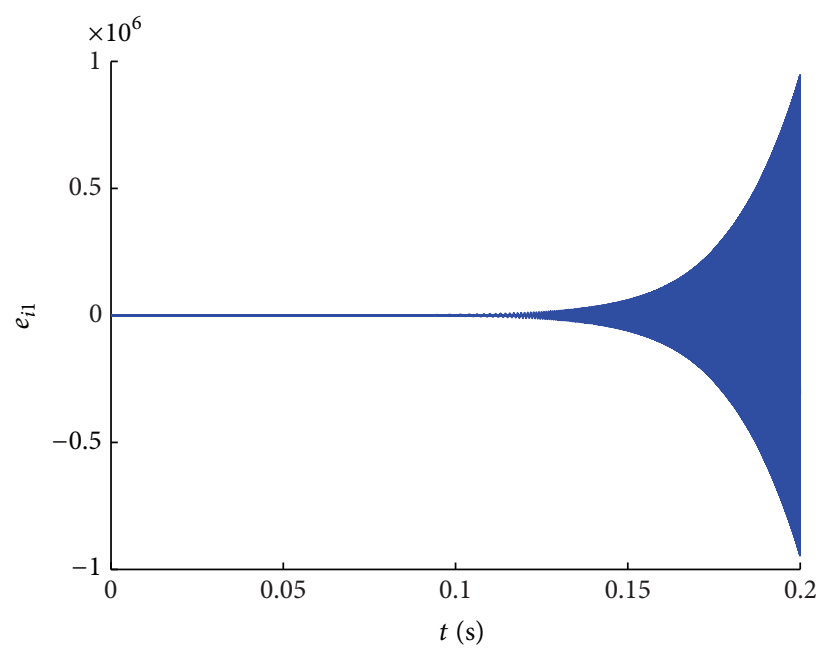

FIGURE 8: The synchronization errors $e_{i 1}$ of the subnetwork 2 of Example 2.

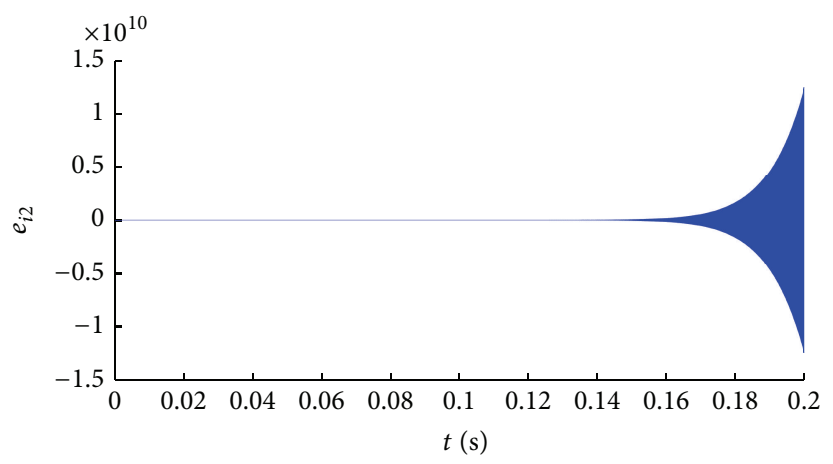

FIgURE 9: The synchronization errors $e_{i 2}$ of the subnetwork 2 of Example 2. 


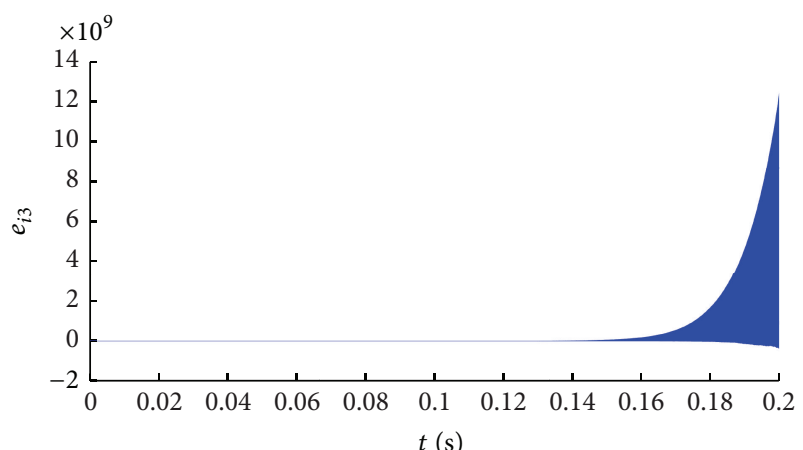

FIGURE 10: The synchronization errors $e_{i 3}$ of the subnetwork 2 of Example 2.

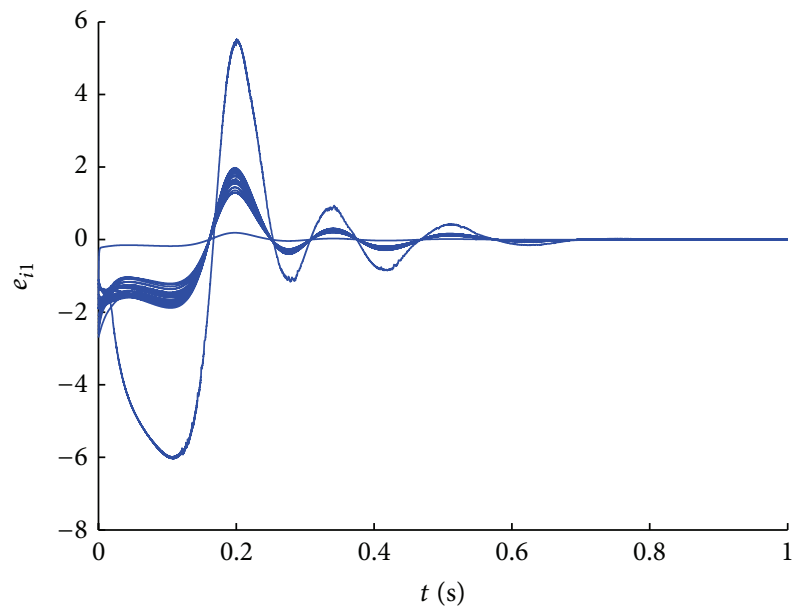

FIGURE 11: The synchronization errors $e_{i 1}$ of the switched networks in Example 2.

From Figures 5 to 10, it is easy to see that two pinning controlled subnetworks cannot achieve synchronization. Applying Theorem 5, we can get synchronization via the designed switching law:

$\sigma(t)$

$= \begin{cases}1, & \text { if } e^{T}\left[\theta I_{30} \otimes \Gamma+c\left(A_{1} \otimes P \Gamma\right)-c\left(Q_{1} \otimes P \Gamma\right)\right] e \leq 0, \\ 2, & \text { if } e^{T}\left[\theta I_{30} \otimes \Gamma+c\left(A_{2} \otimes P \Gamma\right)-c\left(Q_{2} \otimes P \Gamma\right)\right] e \leq 0 .\end{cases}$

The simulations are shown in Figures 11, 12, 13, and 14.

Form Figures 11 to 13, it is easy to see that the states of error systems are very large at the initial time, but after a while, it converges to zero quickly, so the switched complex networks achieved synchronization under the designed switching laws, which verify the validity of Theorem 5 . In Figure 14, the value of $\sigma(t)$ denotes the activated subsystem; it means that if $\sigma(t)=$ 1 , the first subnetwork is activated, and if $\sigma(t)=2$, the second subnetwork is activated.

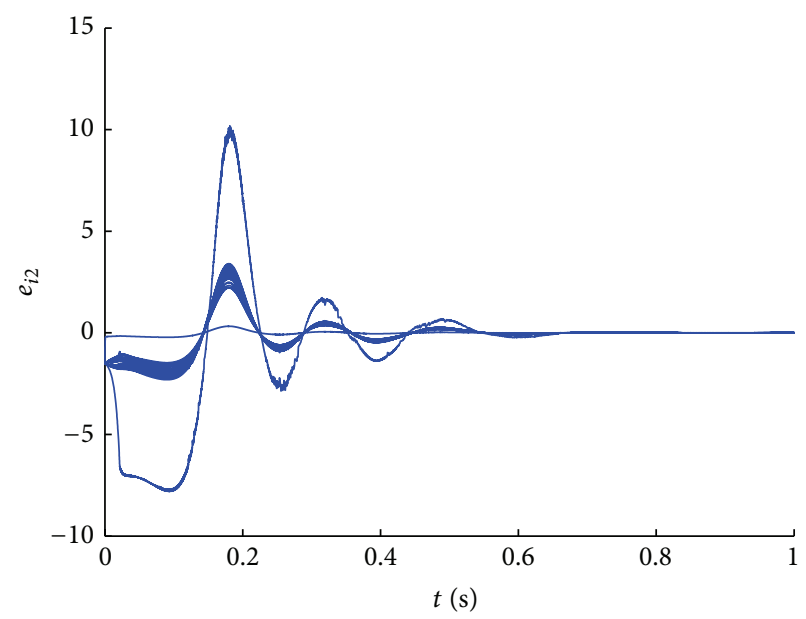

FIGURE 12: The synchronization errors $e_{i 2}$ of the switched networks in Example 2.

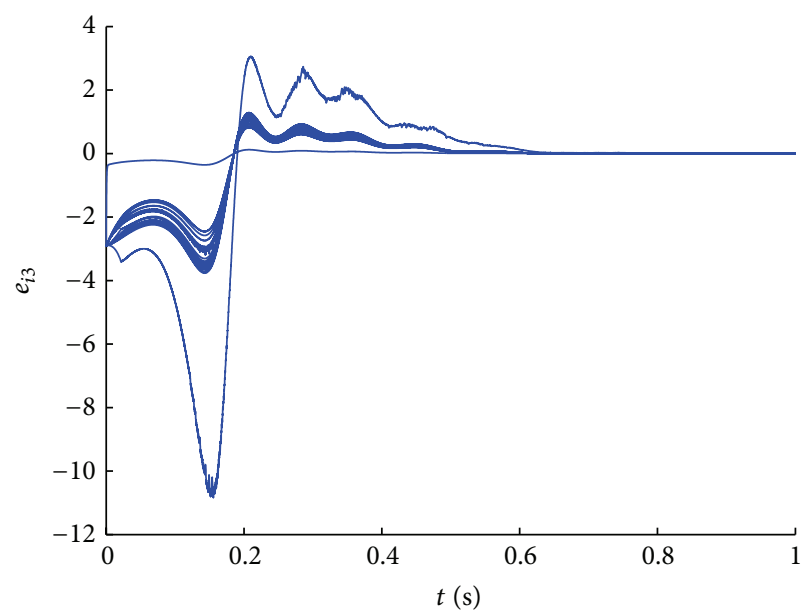

FIGURE 13: The synchronization errors $e_{i 3}$ of the switched networks in Example 2.

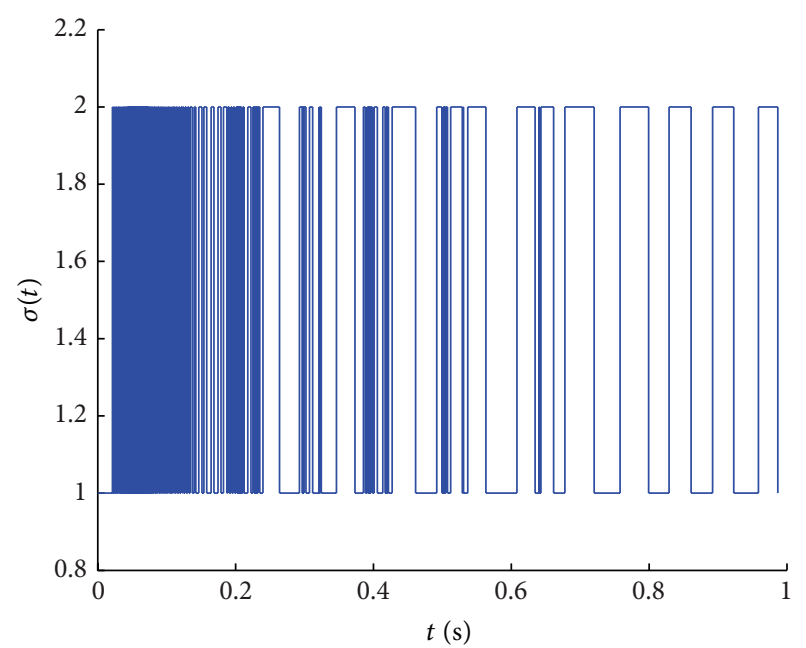

FIgURE 14: Switching signal of Example 2. 


\section{Conclusion}

The pining synchronization problem of a complex network with switching topology is investigated in this paper. Employing common Lyapunov function method and single Lyapunov function method, some criteria are given to ensure the controlled complex networks achieve global pinning synchronization under arbitrary switching and some designed switching law, respectively. The criteria are simple and easy to verified, but this paper only discussed the pinning synchronization problem of switched dynamical networks by common Lyapunov function and single Lyapunov function method. How to solve this problem by multiple Lyapunov function method is a challenging problem which deserves future study.

\section{Conflict of Interests}

The authors declare that there is no conflict of interests regarding the publication of this paper.

\section{Acknowledgments}

This project was supported by the National Natural Science Foundation of China (Grant no. 61272253) and the Programs Foundation of Technology Bureau of Shenyang (Grant no. F12-277-1-08).

\section{References}

[1] D. J. Watts and S. H. Strogatz, "Collective dynamics of "smallworld” networks," Nature, vol. 393, no. 6684, pp. 440-442, 1998.

[2] S. Boccaletti, V. Latora, Y. Moreno, M. Chavez, and D.-U. Hwang, "Complex networks: structure and dynamics," Physics Reports, vol. 424, no. 4-5, pp. 175-308, 2006.

[3] S. H. Strogatz, "Exploring complex networks," Nature, vol. 410, no. 6825 , pp. 268-276, 2001.

[4] J. Lü and G. Chen, "A brief overview of some recent advances in complex dynamical networks control and synchronization," in Proceedings of the IEEE International Symposium on Circuits and Systems (ISCAS '08), pp. 2518-2521, Seattle, Wash, USA, May 2008.

[5] A. Arenas, A. Díaz-Guilera, J. Kurths, Y. Moreno, and C. Zhou, "Synchronization in complex networks," Physics Reports A, vol. 469, no. 3, pp. 93-153, 2008.

[6] L. M. Pecora and T. L. Carroll, "Master stability functions for synchronized coupled systems," Physical Review Letters, vol. 80, no. 10, pp. 2109-2112, 1998.

[7] J. Lu and D. W. C. Ho, "Local and global synchronization in general complex dynamical networks with delay coupling," Chaos, Solitons and Fractals, vol. 37, no. 5, pp. 1497-1510, 2008.

[8] L. Guo, X.-H. Nian, and H. Pan, "Synchronization criteria for general coupling complex networks with time varying delay," Control Theory and Applications, vol. 28, no. 1, pp. 73-78, 2011.

[9] V. N. Belykh, I. V. Belykh, and M. Hasler, "Connection graph stability method for synchronized coupled chaotic systems," Physica D: Nonlinear Phenomena, vol. 195, no. 1-2, pp. 159-187, 2004.
[10] B. Liu, X. Liu, G. Chen, and H. Wang, "Robust impulsive synchronization of uncertain dynamical networks," IEEE Transactions on Circuits and Systems. I. Regular Papers, vol. 52, no. 7, pp. 1431-1441, 2005.

[11] J. Zhou, J. Lu, and J. Lü, "Adaptive synchronization of an uncertain complex dynamical network," IEEE Transactions on Automatic Control, vol. 51, no. 4, pp. 652-656, 2006.

[12] W. W. Yu and J. D. Cao, "Adaptive synchronization and lag synchronization of uncertain dynamical system with time delay based on parameter identification," Physica A, vol. 375, no. 2, pp. 467-482, 2007.

[13] R. O. Grigoriev, M. C. Cross, and H. G. Schuster, "Pinning control of spatiotemporal chaos," Physical Review Letters, vol. 79, no. 15, pp. 2795-2798, 1997.

[14] N. Parekh, S. Parthasarathy, and S. Sinha, "Global and local control of spatiotemporal chaos in coupled map lattices," Physical Review Letters, vol. 81, no. 7, pp. 1401-1404, 1998.

[15] X. F. Wang and G. Chen, "Pinning control of scale-free dynamical networks," Physica A: Statistical Mechanics and Its Applications, vol. 310, no. 3-4, pp. 521-531, 2002.

[16] X. Li, X. Wang, and G. Chen, "Pinning a complex dynamical network to its equilibrium," IEEE Transactions on Circuits and Systems I: Regular Papers, vol. 51, no. 10, pp. 2074-2087, 2004.

[17] F. Sorrentino, M. di Bernardo, F. Garofalo, and G. Chen, "Controllability of complex networks via pinning," Physical Review E, vol. 75, no. 4, Article ID 046103, 6 pages, 2007.

[18] T. Chen, X. Liu, and W. Lu, "Pinning complex networks by a single controller," IEEE Transactions on Circuits and Systems I, vol. 54, no. 6, pp. 1317-1326, 2007.

[19] W. Yu, G. Chen, and J. Lü, "On pinning synchronization of complex dynamical networks," Automatica, vol. 45, no. 2, pp. 429-435, 2009.

[20] J. Zhou, J.-A. Lu, and J. Lü, "Pinning adaptive synchronization of a general complex dynamical network," Automatica, vol. 44, no. 4, pp. 996-1003, 2008.

[21] F. Xiao and L. Wang, "State consensus for multi-agent systems with switching topologies and time-varying delays," International Journal of Control, vol. 79, no. 10, pp. 1277-1284, 2006.

[22] D. J. Hill and G. Chen, "Power systems as dynamic networks," in Proceedings of the IEEE International Symposium on Circuits and Systems, pp. 722-725, May 2006.

[23] D. Liberzon, Switching in Systems and Control, Birkhäuser, Boston, Mass, USA, 2003.

[24] J. Yao, D. J. Hill, Z.-H. Guan, and H. O. Wang, "Synchronization of complex dynamical networks with switching topology via adaptive control," in Proceedings of the 45th IEEE Conference on Decision and Control (CDC '06), pp. 2819-2824, December 2006.

[25] J. Zhao, D. J. Hill, and T. Liu, "Synchronization of complex dynamical networks with switching topology: a switched system point of view," Automatica, vol. 45, no. 11, pp. 2502-2511, 2009.

[26] K. Sehjeong and J. H. David, "Synchronization of a complex network with switched coupling," in Proceedings of the 17th World Congress the International Federation of Automatic Control, pp. 1559-1564, 2008.

[27] L. M. Du and J. Zhao, "A synchronization criterion for dynamical networks with non-identical nodes and switching topology," Control Theory and Applications, vol. 30, no. 5, pp. 649-655, 2013. 


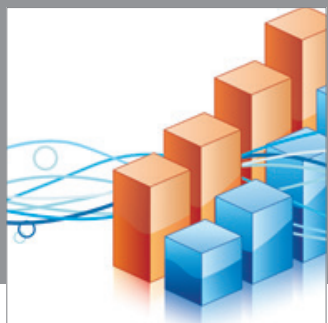

Advances in

Operations Research

mansans

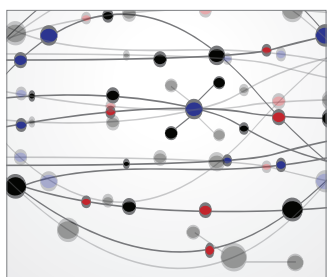

The Scientific World Journal
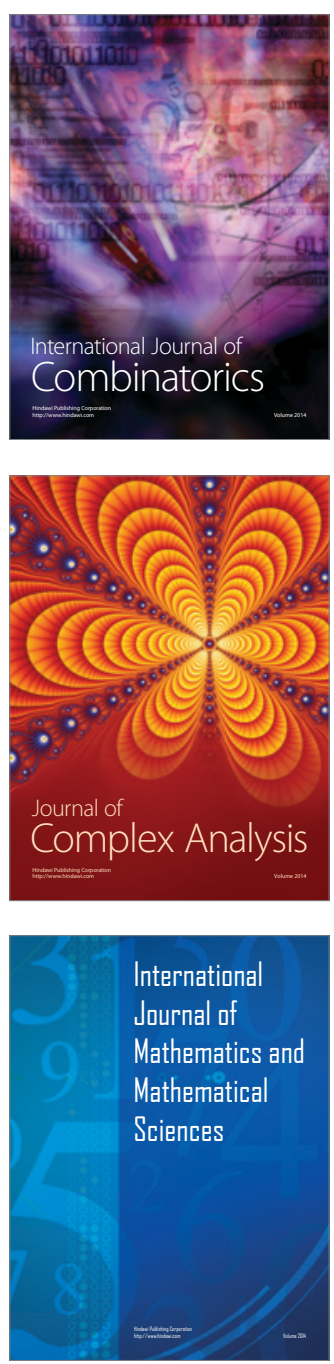
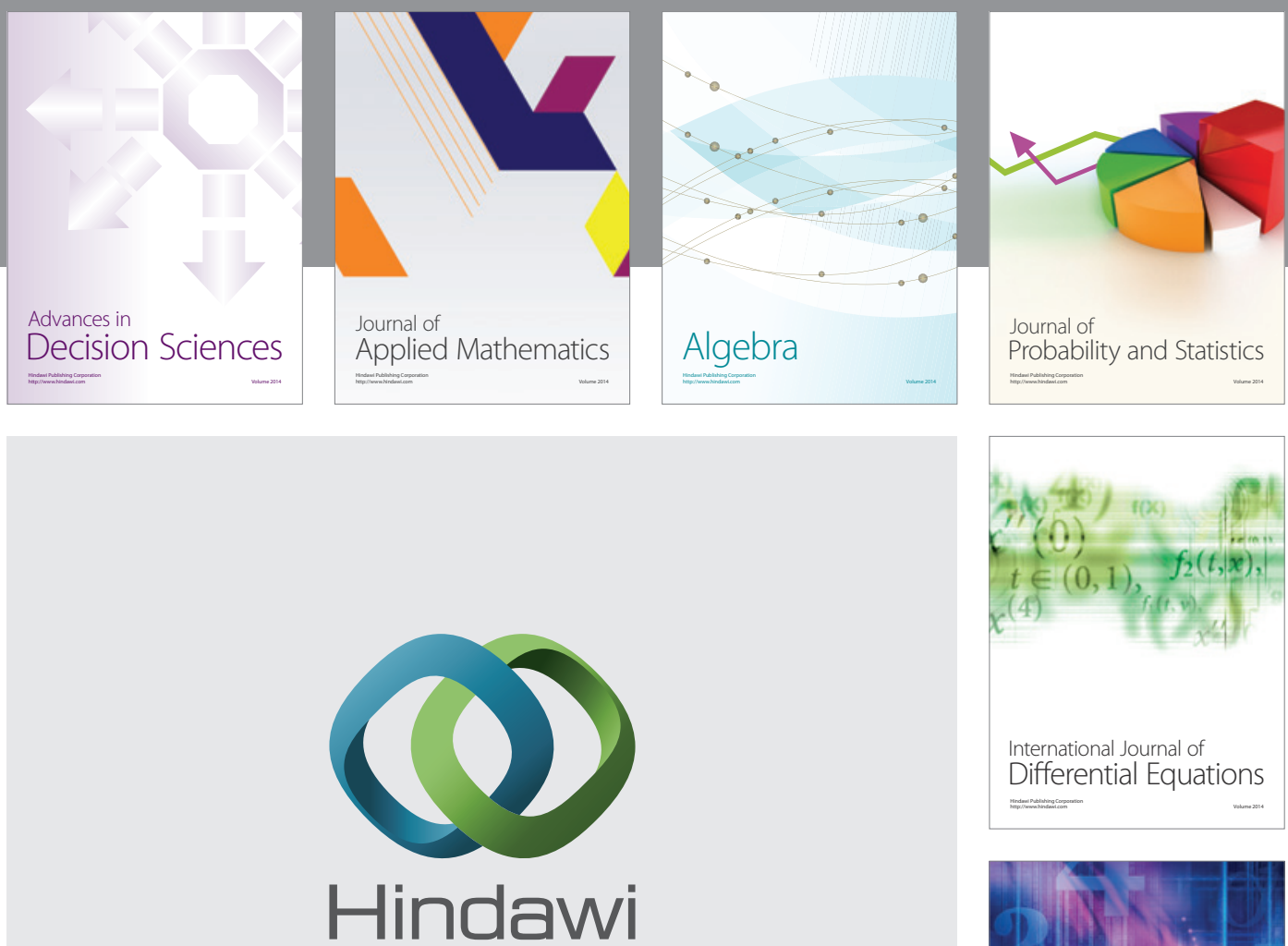

Submit your manuscripts at http://www.hindawi.com
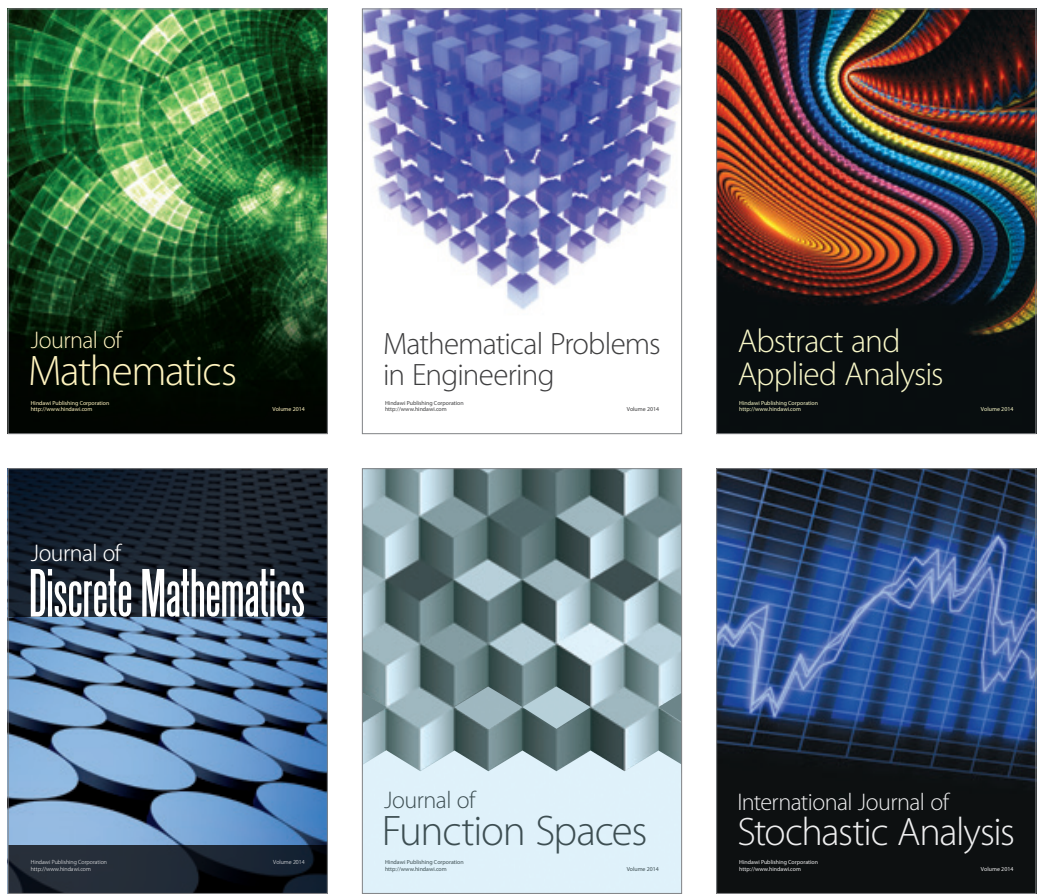

Journal of

Function Spaces

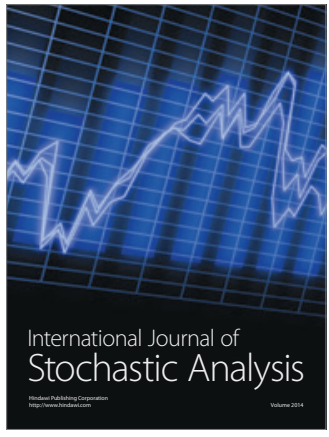

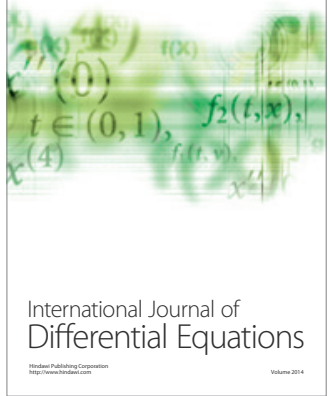
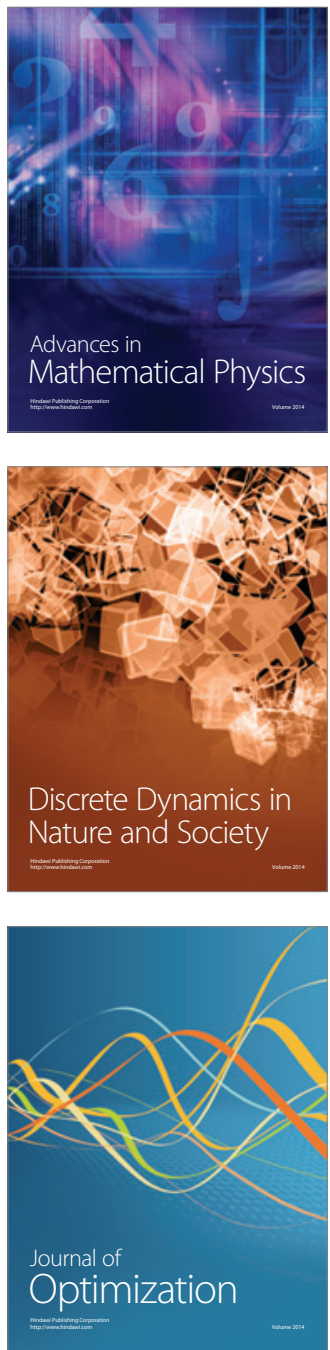